\title{
Ash influence on the ethyl levulinate production from sugarcane molasses mediated by taurine hydrogen sulfate
}

Gustavo Rodrigues Gomes ${ }^{\mathrm{a}}$, Márcia Cristina Breitkreitz ${ }^{\mathrm{a}}$, Julio Cezar Pastre, ${ }^{\mathrm{a}, *}$

a Institute of Chemistry, University of Campinas - UNICAMP, PO Box 6154, 13083-970

Campinas, SP, Brazil. *Corresponding author: jpastre@unicamp.br.

\section{Summary}

\section{List of Figures}

Figure S1. ${ }^{1} \mathrm{H}$ NMR spectrum $\left(250 \mathrm{MHz}, \mathrm{D}_{2} \mathrm{O}\right)$ of taurine hydrogen sulfate. S2

Figure S2. ${ }^{13} \mathrm{C}$ NMR spectrum $\left(62.5 \mathrm{MHz}, \mathrm{D}_{2} \mathrm{O}\right)$ of taurine hydrogen sulfate. ........................... 2

Figure S3. ${ }^{1} \mathrm{H}$ NMR spectrum $\left(250 \mathrm{MHz}, \mathrm{D}_{2} \mathrm{O}\right)$ of EMF.........................................................

Figure S4. ${ }^{13} \mathrm{C}$ NMR spectrum $\left(62.5 \mathrm{MHz}, \mathrm{D}_{2} \mathrm{O}\right)$ of EMF. ............................................... 3

Figure S5. Predicted vs. observed analysis for EL production from fructose.............................S5

Figure S6. Pareto Chart of EMF production from fructose. ..........................................................

Figure S7. Predicted vs. observed analysis for EL production from sucrose.............................S8

Figure S8. GC-MS analysis of EL production of ash-free molasses at different scales.. .........S11

Figure S9. ${ }^{1} \mathrm{H} \mathrm{NMR}\left(250 \mathrm{MHz}, \mathrm{CDCl}_{3}\right)$ and ${ }^{13} \mathrm{C} \mathrm{NMR}$ spectrum $\left(62.5 \mathrm{MHz}, \mathrm{CDCl}_{3}\right)$ of (A) small-scale experiment and (B)10-fold scale-up experiment.. .............................................S12

Figure S10. Taurine hydrogen sulfate recycling eexperiments.............................................S14

\section{List of Tables}

Table S1. ANOVA analysis of the $2^{3}$ full factorial design for EL production from fructose. ....S4 Table S2. ANOVA analysis of the $2^{3}$ full factorial design for EL production from sucrose. .....S7

Table S3. Chemical composition of the molasses inorganic fraction. . 99

Table S4. Scale-up experiments for EL production from treated molasses S10 


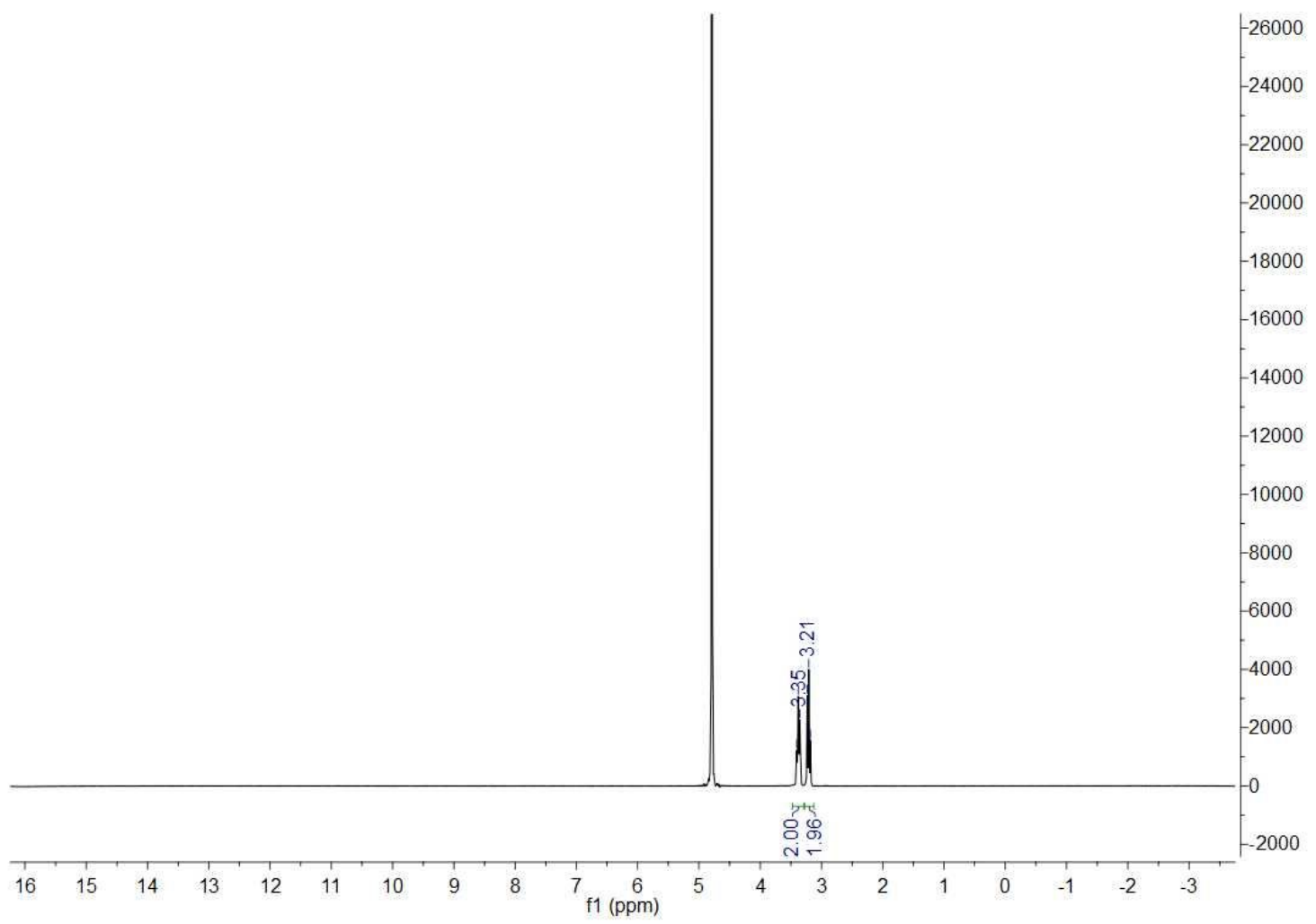

Figure S1. ${ }^{1} \mathrm{H}$ NMR spectrum $\left(250 \mathrm{MHz}, \mathrm{D}_{2} \mathrm{O}\right)$ of taurine hydrogen sulfate.

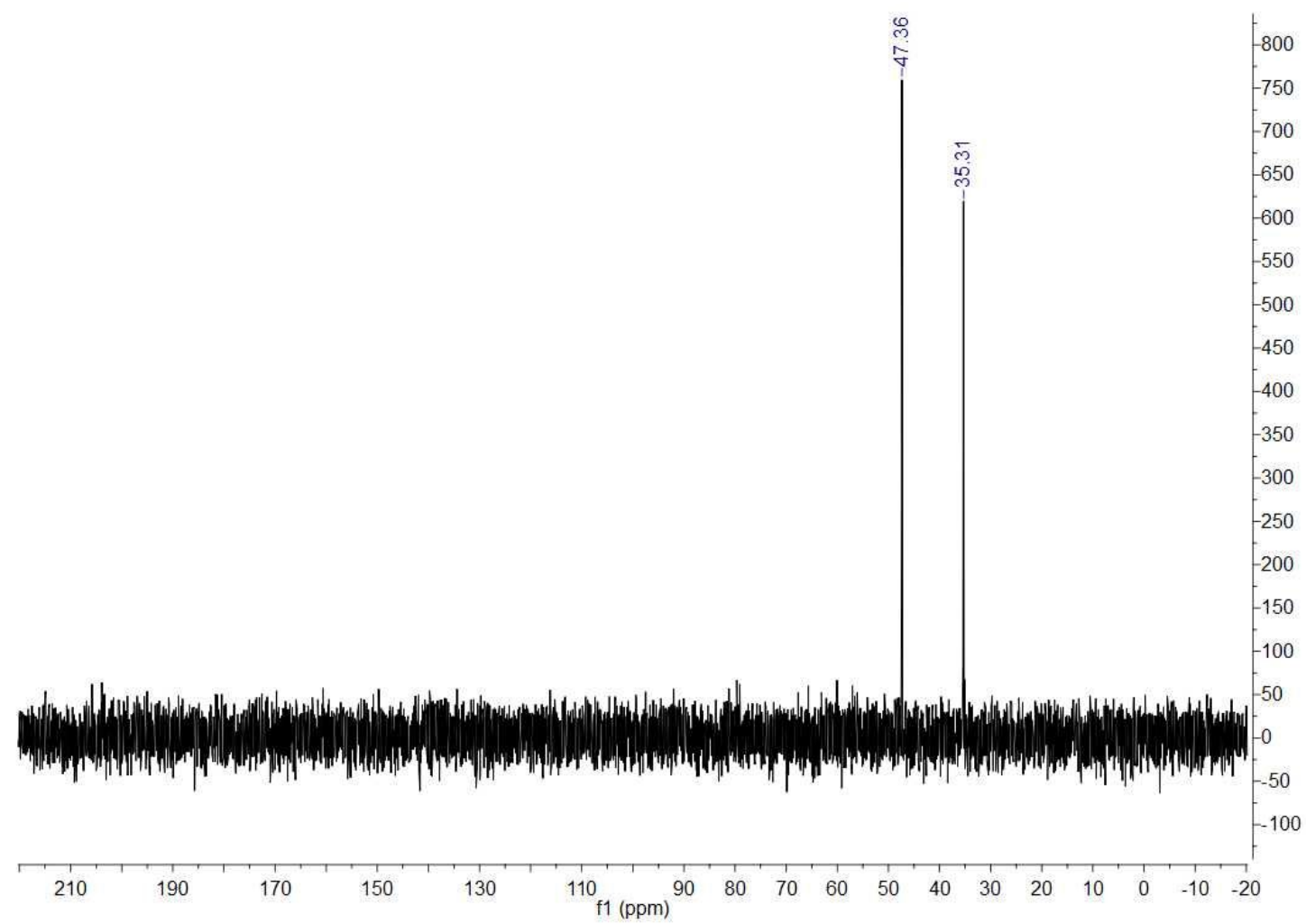

Figure S2. ${ }^{13} \mathrm{C}$ NMR spectrum $\left(62.5 \mathrm{MHz}, \mathrm{D}_{2} \mathrm{O}\right)$ of taurine hydrogen sulfate. 


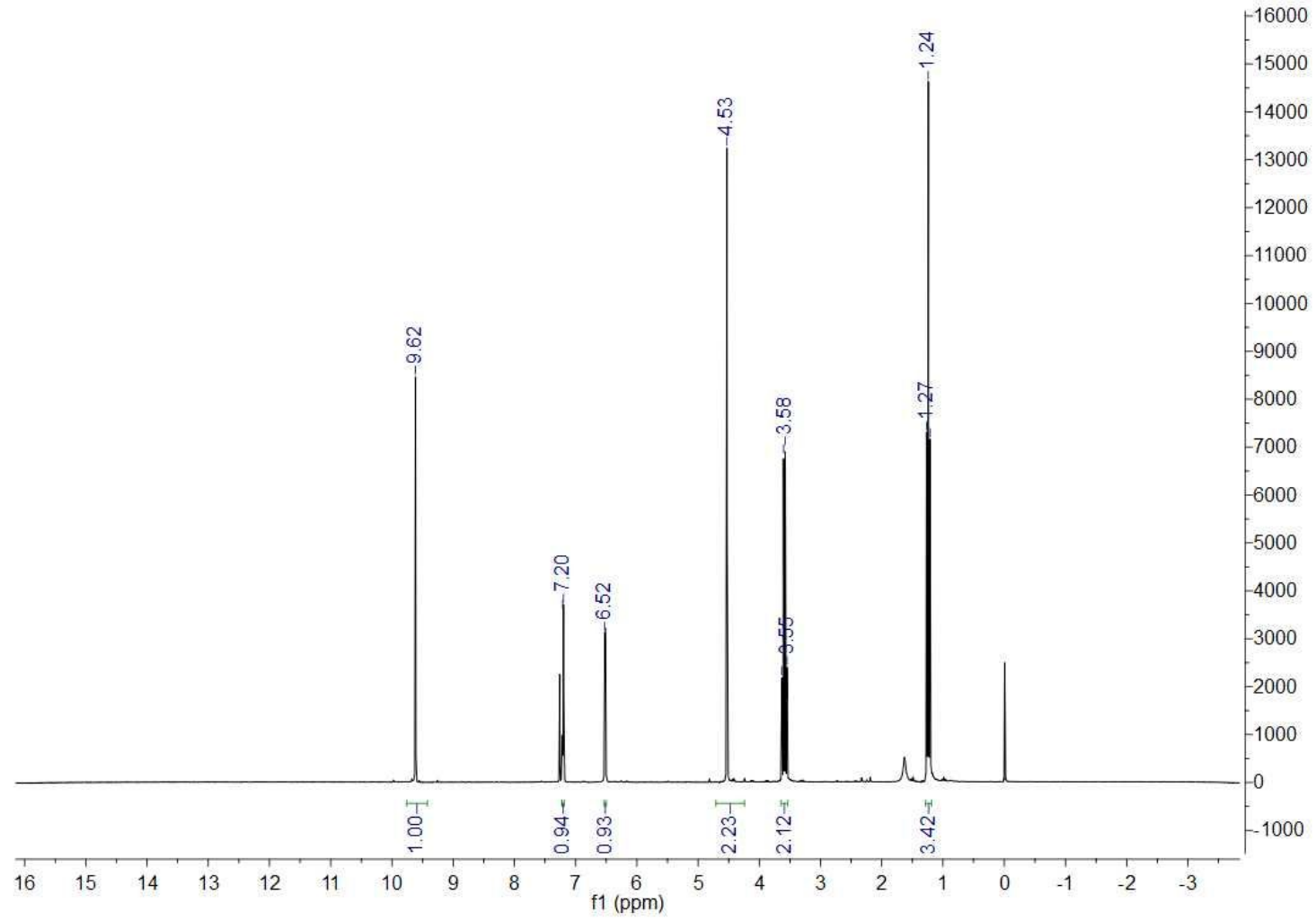

Figure S3. ${ }^{1} \mathrm{H}$ NMR spectrum $\left(250 \mathrm{MHz}, \mathrm{CDCl}_{3}\right)$ of EMF.

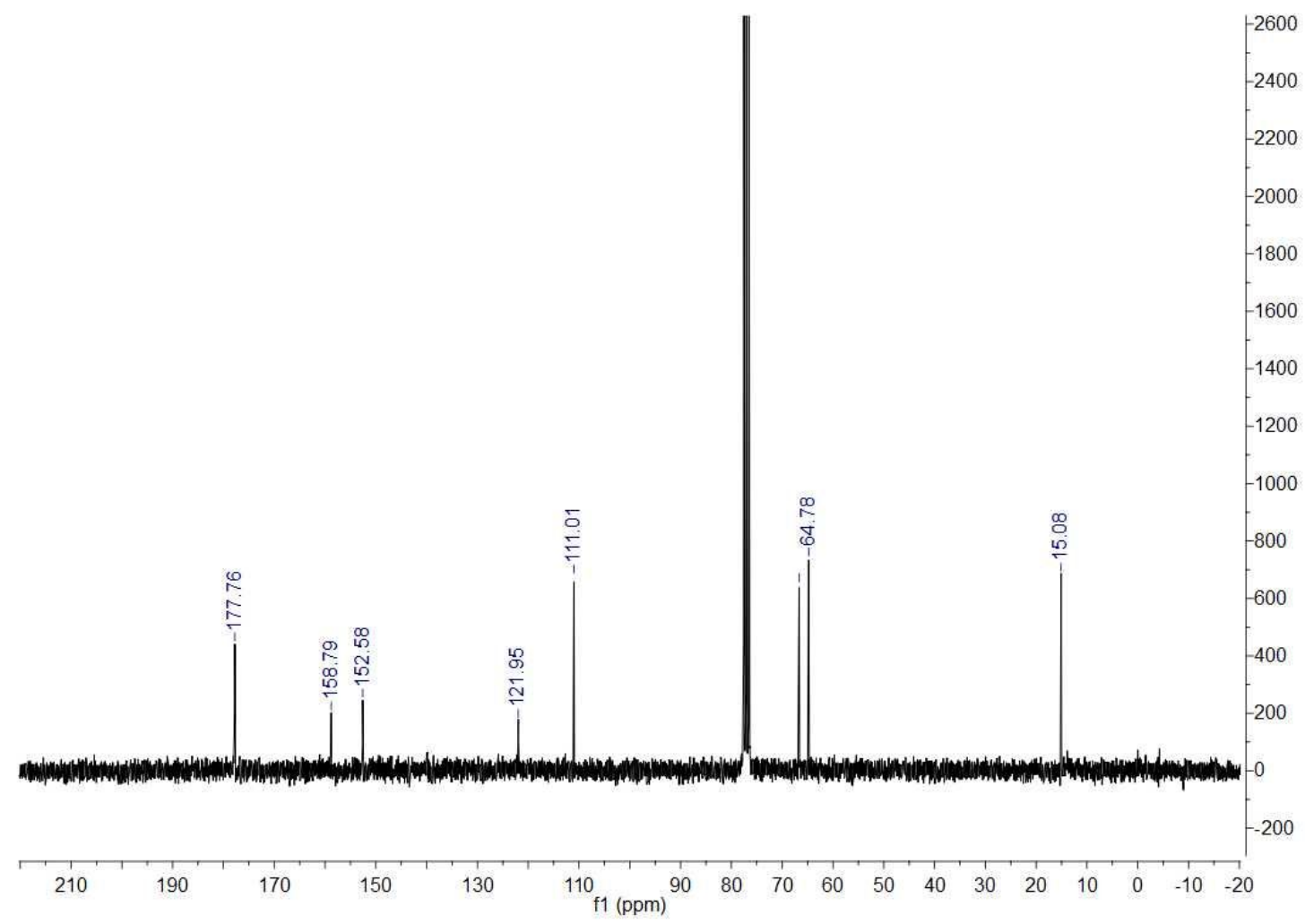

Figure S4. ${ }^{13} \mathrm{C}$ NMR spectrum $\left(62.5 \mathrm{MHz}, \mathrm{CDCl}_{3}\right)$ of EMF. 
Table S1. ANOVA analysis of the $2^{3}$ full factorial design for EL production from fructose.

\begin{tabular}{ccccccc}
\hline Source & $\begin{array}{c}\text { Sum of } \\
\text { Squares }\end{array}$ & df & $\begin{array}{c}\text { Mean } \\
\text { Square }\end{array}$ & $\begin{array}{c}\text { F } \\
\text { Value }\end{array}$ & $\begin{array}{c}\text { p-value } \\
\text { Prob }>\text { F }\end{array}$ & \\
\hline Model & 0.83 & 5 & 0.17 & 68.88 & 0.0001 & significant \\
A-Temperature & 0.29 & 1 & 0.29 & 119.07 & 0.0001 & \\
B-Time & 0.29 & 1 & 0.29 & 119.18 & 0.0001 & \\
C-Catalyst & 0.13 & 1 & 0.13 & 55.40 & 0.0007 & \\
AC & 0.044 & 1 & 0.044 & 18.31 & 0.0079 & \\
BC & 0.078 & 1 & 0.078 & 32.41 & 0.0023 & \\
Residual & 0.012 & 5 & $2.405 \mathrm{E}-003$ & & & \\
Lack of Fit & $9.616 \mathrm{E}-003$ & 3 & $3.205 \mathrm{E}-003$ & 2.66 & 0.2847 & not significant \\
Pure Error & $2.407 \mathrm{E}-003$ & 2 & $1.203 \mathrm{E}-003$ & & & \\
Cor Total & 0.84 & 10 & & & & \\
\hline
\end{tabular}


Design-Expert $\circledast$ Software Yield EL

Color points by value of Yield EL:

П76

6

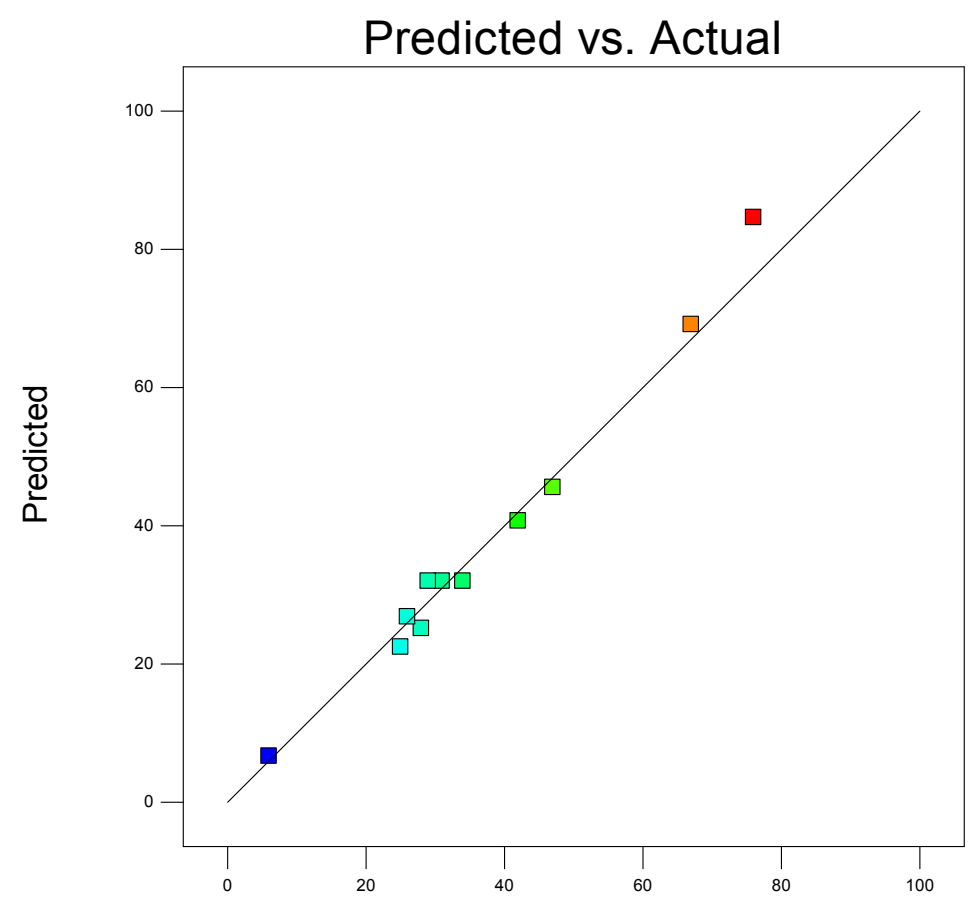

Actual

Figure S5. Predicted vs. observed analysis for EL production from fructose. 


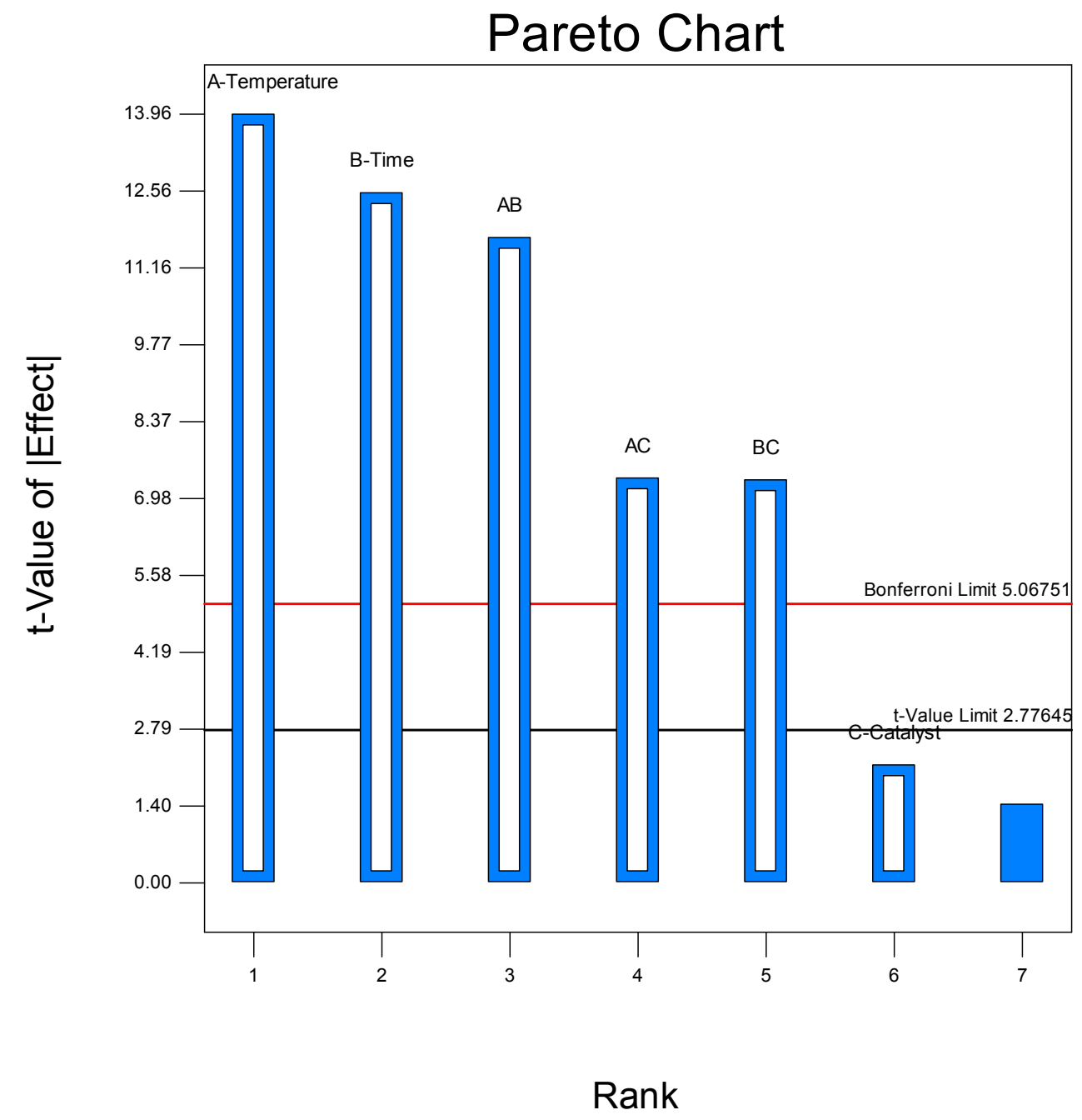

Figure S6. Pareto Chart of EMF production from fructose. 
Table S2. ANOVA analysis of the $2^{3}$ full factorial design for EL production from sucrose.

\begin{tabular}{ccccccc}
\hline Source & $\begin{array}{c}\text { Sum of } \\
\text { Squares }\end{array}$ & df & $\begin{array}{c}\text { Mean } \\
\text { Square }\end{array}$ & $\begin{array}{c}\text { F } \\
\text { Value }\end{array}$ & $\begin{array}{c}\text { p-value } \\
\text { Prob > F }\end{array}$ & \\
\hline Model & 2365.50 & 7 & 337.93 & 71.71 & 0.0025 & significant \\
A-Temperature & 1264.05 & 1 & 1264.05 & 268.25 & 0.0005 & \\
B-Time & 451.25 & 1 & 451.25 & 95.76 & 0.0023 & \\
C-Catalyst & 242.00 & 1 & 242.00 & 51.36 & 0.0056 & \\
AB & 68.45 & 1 & 68.45 & 14.53 & 0.0318 & \\
AC & 4.50 & 1 & 4.50 & 0.95 & 0.4005 & \\
BC & 98.00 & 1 & 98.00 & 20.80 & 0.0198 & \\
ABC & 180.50 & 1 & 180.50 & 38.31 & 0.0085 & \\
Residual & 14.14 & 3 & 4.71 & & & \\
Lack of Fit & 0.14 & 1 & 0.14 & 0.019 & 0.9018 & not significant \\
Pure Error & 14.00 & 2 & 7.00 & & & \\
Cor Total & 2379.64 & 10 & & & & \\
\hline
\end{tabular}


Design-Expert@ Software

EL

Color points by value of

EL: 54

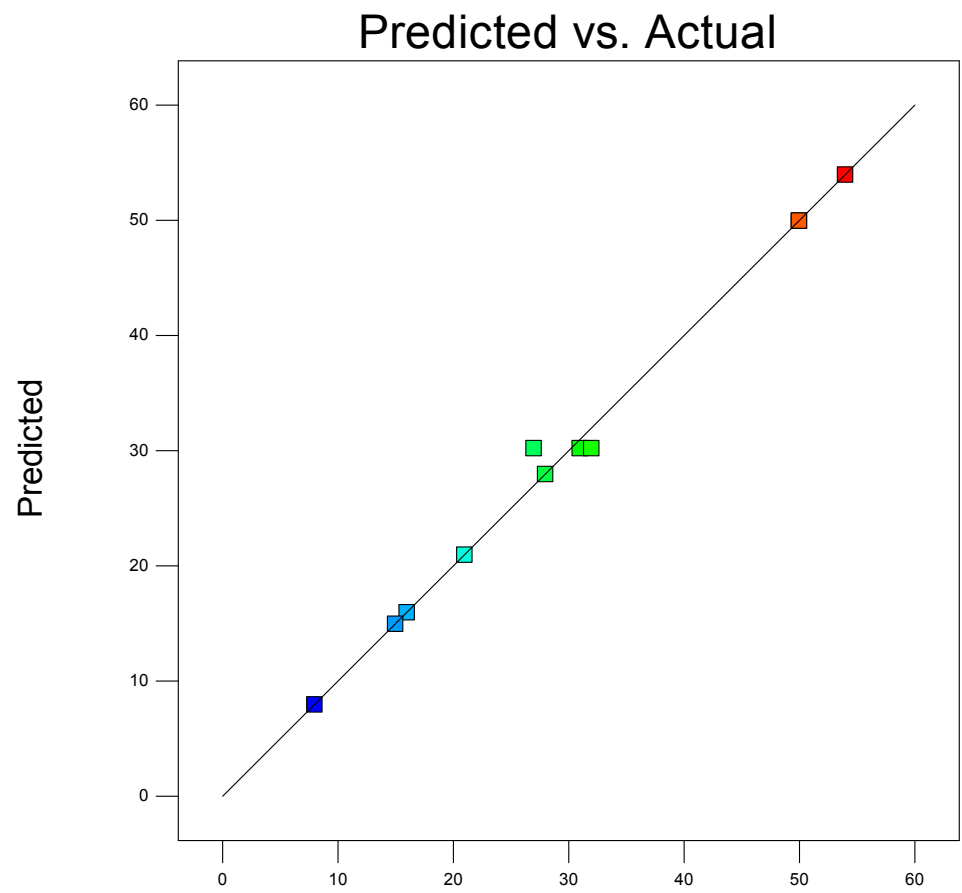

Actual

Figure S7. Predicted vs. observed analysis for EL production from sucrose. 
Table S3. Chemical composition of the molasses inorganic fraction.

\begin{tabular}{ccc}
\hline Element & \% & SD \\
\hline $\mathrm{K}$ & $69.0217 \%$ & $2.0622 \%$ \\
$\mathrm{Ca}$ & $14.4108 \%$ & $3.5510 \%$ \\
$\mathrm{Cl}$ & $11.9533 \%$ & $2.1404 \%$ \\
$\mathrm{~S}$ & $2.0644 \%$ & $0.2021 \%$ \\
$\mathrm{Mg}$ & $0.4213 \%$ & $0.9093 \%$ \\
$\mathrm{Fe}$ & $0.7038 \%$ & $0.3276 \%$ \\
$\mathrm{Cr}$ & $0.3325 \%$ & $0.4578 \%$ \\
$\mathrm{Ni}$ & $0.4609 \%$ & $0.4362 \%$ \\
$\mathrm{Mn}$ & $0.2187 \%$ & $0.0451 \%$ \\
$\mathrm{P}$ & $0.1597 \%$ & $0.0630 \%$ \\
$\mathrm{Cu}$ & $0.2259 \%$ & $0.1528 \%$ \\
$\mathrm{Si}$ & $0.0586 \%$ & $0.0628 \%$ \\
$\mathrm{Zn}$ & $0.0073 \%$ & $0.0230 \%$
\end{tabular}


Table S4. Scale-up experiments for EL production from treated molasses.

\begin{tabular}{cc}
\hline Scale & EL (\%) \\
\hline Small scale $(\mathrm{mg})^{\mathrm{a}}$ & $82 \pm 4$ \\
10 -fold scale -up $(\mathrm{g})^{\mathrm{b}}$ & $58 \pm 3$
\end{tabular}

a Treated molasses: $280 \mathrm{mg}$; Cat. $23 \mathrm{mg}$; EtOH: $2 \mathrm{~mL}$; T: $180^{\circ} \mathrm{C}$; t: $2 \mathrm{~h}$; MW

b Treated molasses: 2.80 g; Cat. $230 \mathrm{mg}$; EtOH: $20 \mathrm{~mL}$; T: $180^{\circ} \mathrm{C}$; t: $2 \mathrm{~h}$; MW 


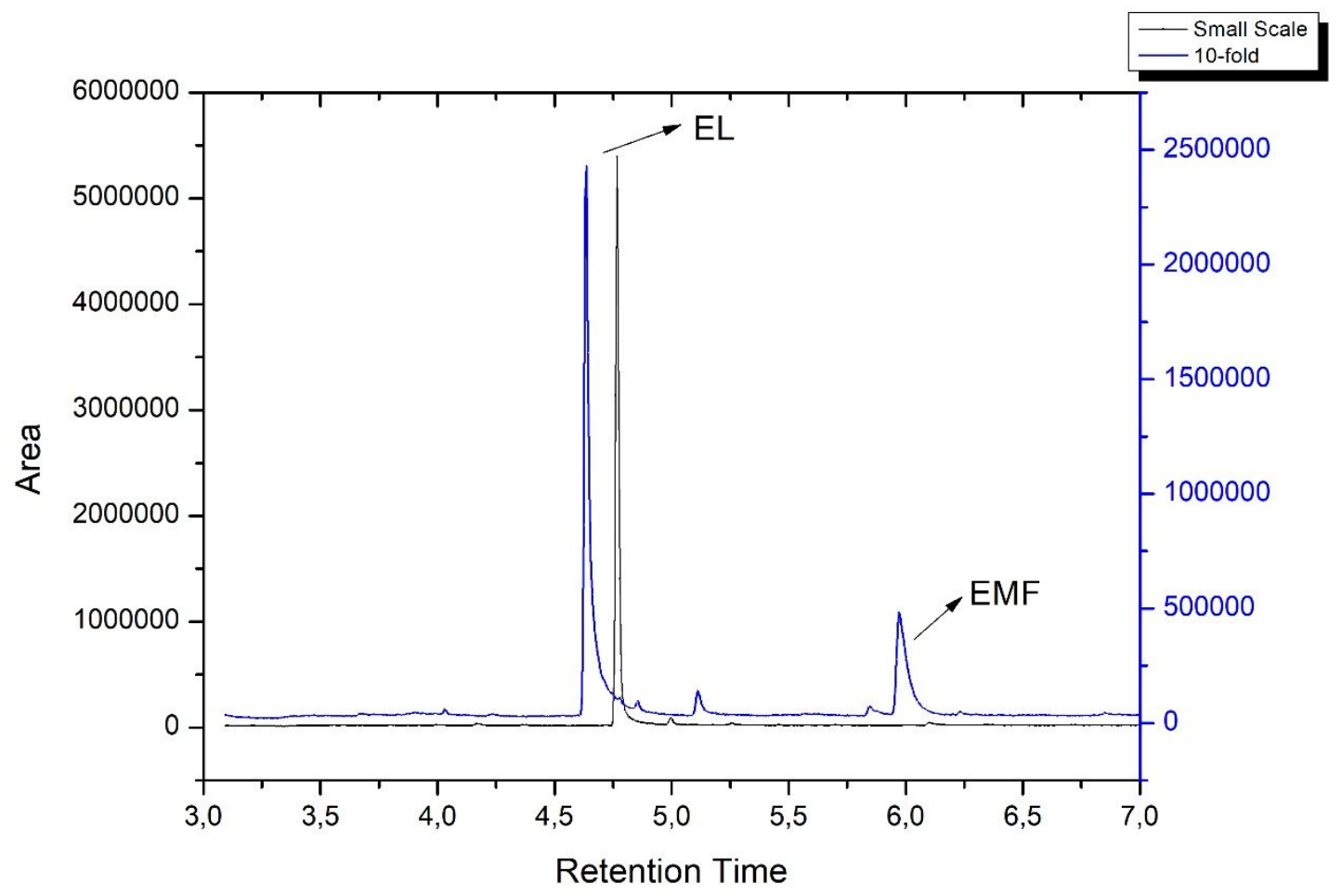

Figure S8. GC-MS analysis of EL production of ash-free molasses at different scales. 
A

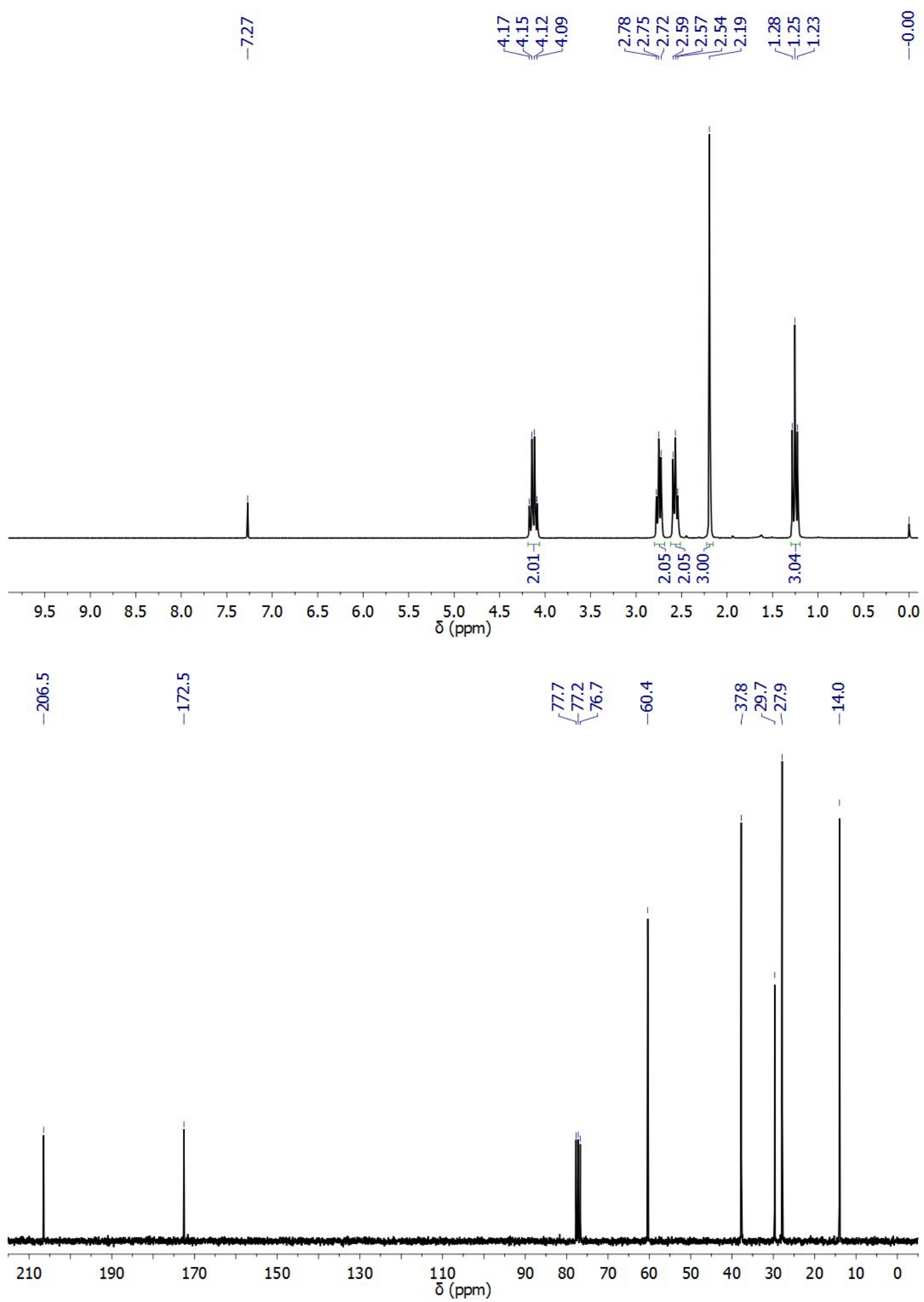


B

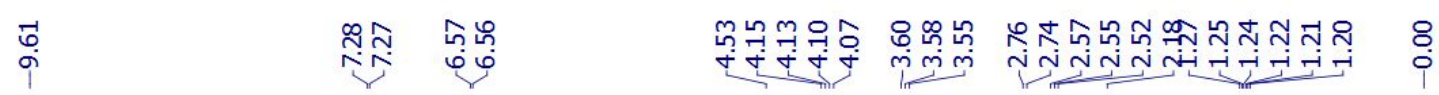

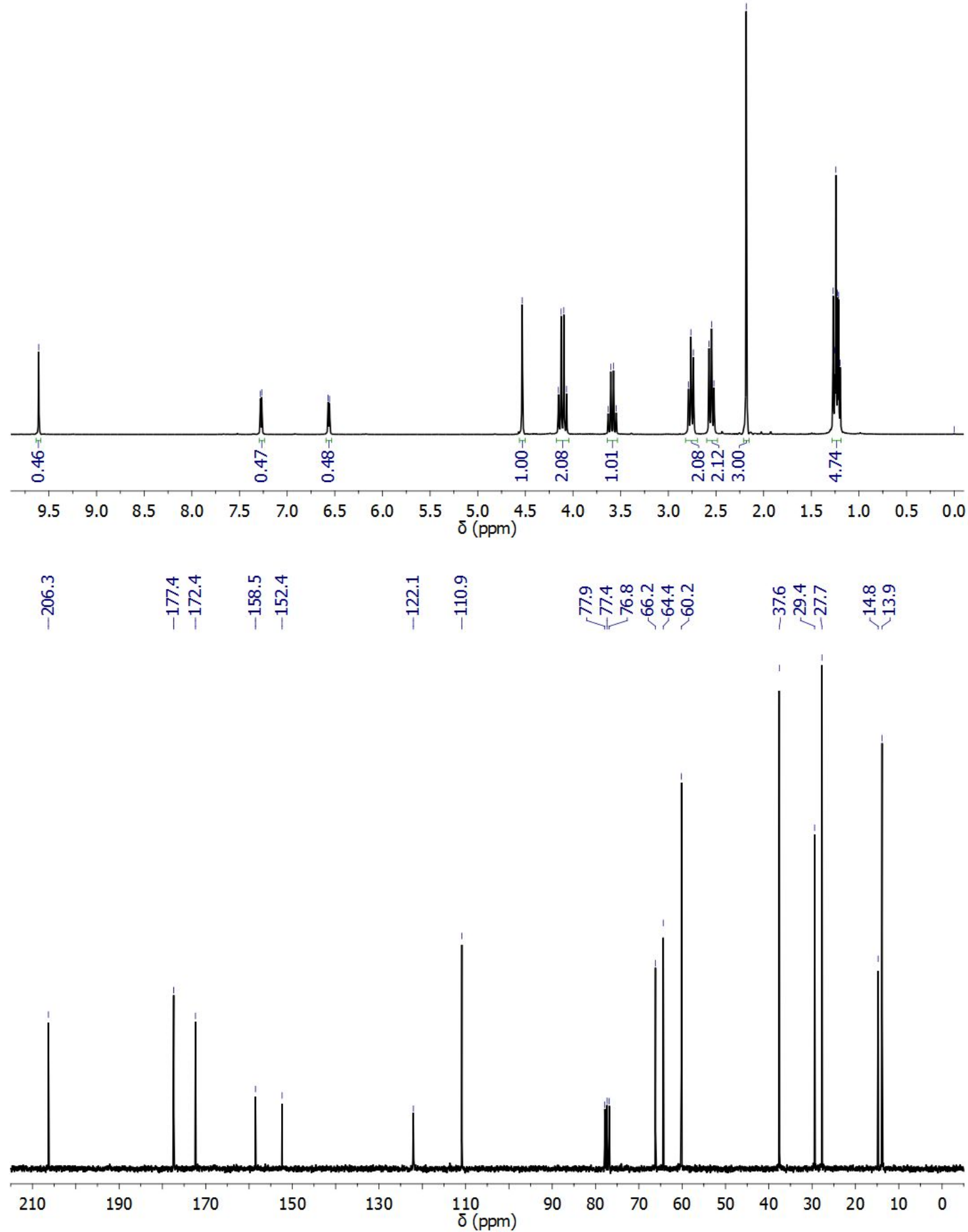

Figure S9. ${ }^{1} \mathrm{H}$ NMR $\left(250 \mathrm{MHz}, \mathrm{CDCl}_{3}\right)$ and ${ }^{13} \mathrm{C} \mathrm{NMR}$ spectrum $\left(62.5 \mathrm{MHz}, \mathrm{CDCl}_{3}\right)$ of (A) small-scale experiment and (B) 10-fold scale-up experiment. 


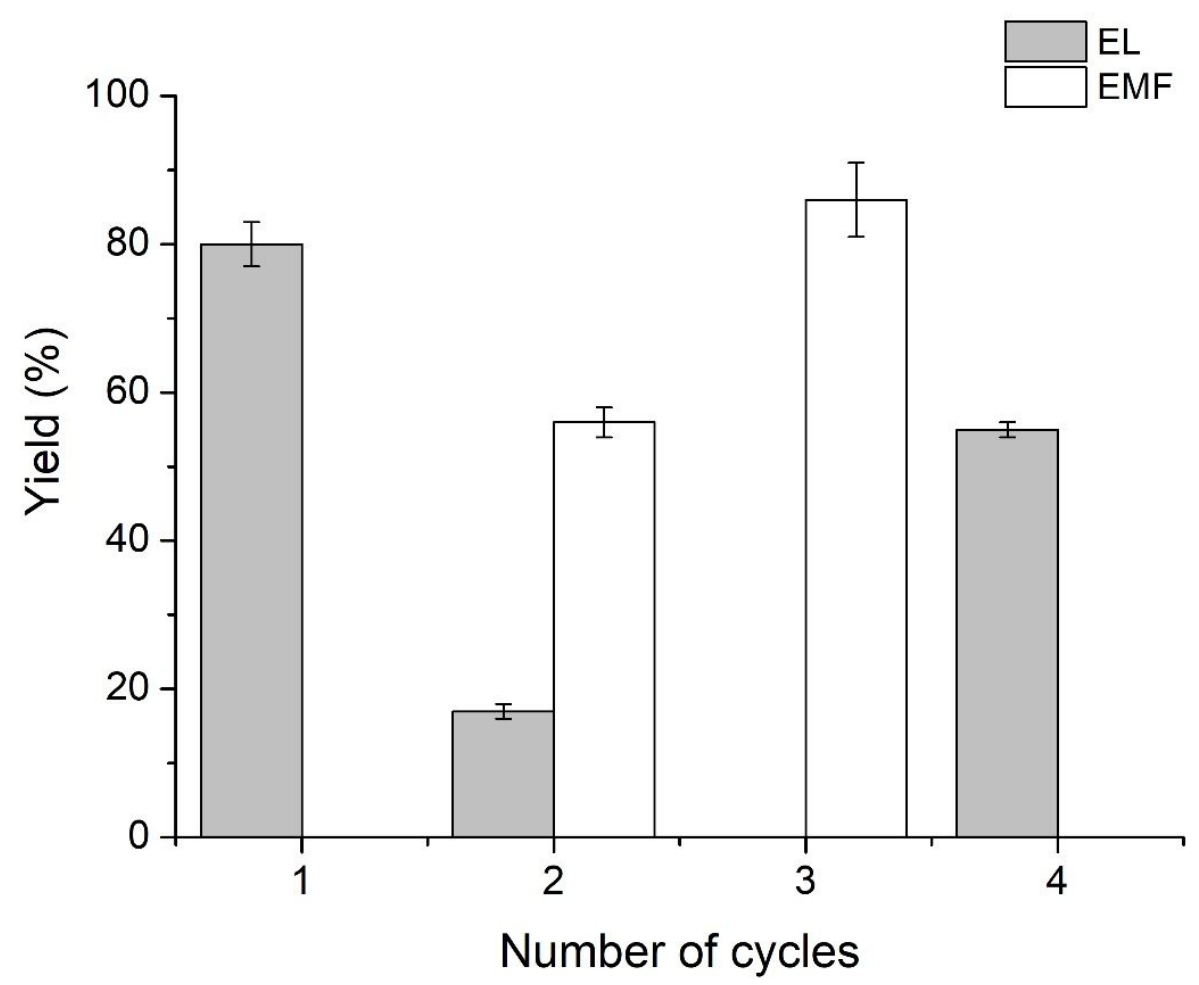

Figure S10. Taurine hydrogen sulfate recycling experiments. 